\title{
Erratum to "Erratum: Mortality prediction after cardiac surgery: blood lactate is indispensible". Thorac Cardiovasc Surg 2013; 61(4): 375-376
}

\author{
Akmal M. A. Badreldin ${ }^{1,2}$ Fabian Doerr ${ }^{3}$ Sherif Elsobky ${ }^{4}$ Bernhard R. Brehm ${ }^{2}$ Mohamed Abul-dahab ${ }^{5}$ \\ Thomas Lehmann $^{6}$ Ole Bayer ${ }^{7}$ Thorsten Wahlers ${ }^{8}$ Khosro Hekmat ${ }^{1,8}$
}

${ }^{1}$ Cardiothoracic Surgery, CardioClinic, Cologne, NRW, Germany

2 Department of Cardiology, Catholic Hospital Koblenz-Marienhof, Koblenz, Germany

3 Faculty of Medicine, Friedrich-Schiller-University of Jena, Erlanger Address for correspondence Akmal M. A. Badreldin, MD, OscarAbisch-Weg 34, 51145 Cologne, Germany

Allee 101, Jena, Germany

${ }^{4}$ Wolfson Medical School, University of Glasgow, University Avenue, Glasgow, United Kingdom

${ }^{5}$ Department of Cardiothoracic Surgery, Kasr-El-Aini School of Medicine, Cairo University, Egypt

${ }^{6}$ Department of Medical Statistics, Computer Sciences and Documentation, Friedrich-Schiller-University of Jena, Jena, Germany

7 Department of Anesthesiology and Intensive Care Medicine,

Friedrich-Schiller-University of Jena, Jena, Germany

8 Department of Cardiothoracic Surgery, University of Cologne,

Cologne, Germany

Thorac Cardiovasc Surg 2014;62:273-274.

\section{ERRATUM}

It has been brought to the Editor-in-Chief's attention that in conjunction with the first Erratum the co-author Sherif Elsobky was inadvertently omitted.

Original article published in issue 61(8); DOI 10.1055/s-0032-1324796.

First erratum published in issue 61(4); DOI 10.1055/s-0033-1343387.

The definitive and correct list of authors is as follows:

Akmal M. A. Badreldin, Fabian Doerr, Sherif Elsobky, Bernhard R. Brehm, Mohamed Abuldahab, Thomas Lehmann, Ole Bayer, Thorsten Wahlers, Khosro Hekmat

Markus K. Heinemann, MD, PhD

Editor-in-Chief

The Thoracic and Cardiovascular Surgeon

Editor-in-Chief's Commentary It is a sad fact that a fair and extremely unsatisfactory portion of an Editor's administrative work has to do with authorship conflicts. It should be recalled that in the course of a serious scientific publication the list of authors is to be defined at the outset and must not grow or be changed during its creation. Of course, we are all human and errors may occur. From an Editor's perspective I must, however, urge all our authors to apply the same diligence to their writing as they (hopefully) did to their research. The Thoracic and Cardiovascular Surgeon has introduced the mandatory Conditions for Publication form. Among other things, it is also meant to help avoiding ill-defined authorships in the future. Unfortunately it seems to be a necessary step for orientation in scientific publishing, a field otherwise known as Vanity Fair. (c) 2014 Georg Thieme Verlag KG Stuttgart · New York
DOI http://dx.doi.org/ 10.1055/s-0034-1372419. ISSN 0171-6425. 